Research Article

\title{
Sufficient Conditions and Duality Theorems for Nondifferentiable Minimax Fractional Programming
}

\author{
Shun-Chin Ho \\ Center of General Education, Chung Jen College of Nursing, Health Science and Management, \\ Dalin 62241, Taiwan \\ Correspondence should be addressed to Shun-Chin Ho, scho31@gmail.com
}

Received 20 October 2010; Accepted 28 November 2010

Academic Editors: R. Barrio and S. Zhang

Copyright (C) 2011 Shun-Chin Ho. This is an open access article distributed under the Creative Commons Attribution License, which permits unrestricted use, distribution, and reproduction in any medium, provided the original work is properly cited.

\begin{abstract}
We consider nondifferentiable minimax fractional programming problems involving $B$ - $(p, r)$-invex functions with respect to $\eta$ and $b$. Sufficient optimality conditions and duality results for a class of nondifferentiable minimax fractional programming problems are obtained undr $B$ - $(p, r)$-invexity assumption on objective and constraint functions. Parametric duality, Mond-Weir duality, and Wolfe duality problems may be formulated, and duality results are derived under $B-(p, r)$-invex functions.
\end{abstract}

\section{Introduction}

Convexity plays an important role in many aspects of mathematical programming including sufficient optimality conditions and duality theorems. In general, we use the invex function to replace convexity on sufficient optimality conditions and duality theorems (see, e.g., [1-6]).

Many authors investigated the optimality conditions and duality theorems for minimax (fractional) programming problems. For details, one can consult [1-14]. In particular, Lai et al. [10] have established the theorems of necessary and sufficient optimality conditions for nondifferentiable minimax fractional problem under the conditions of convexity. In [11], Lai and Lee employed the optimality conditions to construct two parameter-free dual models of nondifferentiable minimax fractional programming problem which involve pseudoconvex and quasiconvex functions, and derived weak and strong duality theorems. In the formulation of the dual models in [11] optimality conditions given in [10] are used. Mishra et al. [4] derived a Kuhn-Tucker-type sufficient optimality condition for an optimal solution to the nondifferentiable minimax fractional programming problem and established weak, strong, and converse duality theorems for the problem and its three 
different forms of dual problems under generalized univexity. Mishra et al. [5, 13] considered the nondifferentiable minimax fractional programming problem and obtain optimality and duality results under generalized $\alpha$-invexity [5] and generalized $\alpha$-unvexity [13]. Recently, Antczak [15] defined a new class of functions, named $B-(p, r)$-invex, which is an extension of invex function. In [1], parametric and nonparametric sufficient optimality conditions and several parametric and parameter-free duality models for the generalized fractional minimax programs are obtained under $B-(p, r)$-invexity assumption on objective and constraint functions.

In this paper, we are inspired to extend the result of Lai et al. [10] to $B-(p, r)$-invexity and organize this paper as follows. In Section 2 we introduce some basic results. We establish sufficient optimality conditions for nondifferentiable minimax fractional programming problem under $B-(p, r)$-invex with respect to the same function $\eta$ and with respect to, not necessarily, the same function $b$ in Section 3. Employing these results, we construct three dual problems in Sections 4-6. Here we investigate weak, strong, and strict converse duality theorems under the framework of $B-(p, r)$-invex with respect to the same function $\eta$ and with respect to, not necessarily, the same function $b$.

\section{Some Notations and Preliminary Results}

Let $\mathbb{R}^{n}$ be the $n$-dimensional Euclidean space and $\mathbb{R}_{+}^{n}$ its nonnegative orthant. Throughout the paper, let $X$ be a nonempty open set of $\mathbb{R}^{\ltimes}$.

The following definition can be found in [15].

Definition 2.1 (see [15]). Let $p$ and $r$ be any real numbers. The differentiable function $f: X \rightarrow$ $\mathbb{R}$ is said to be (strictly) $B$ - $(p, r)$-invex with respect to $\eta$ and $b$ at $u \in X$ on a nonempty set $X \subset \mathbb{R}^{n}$ if, there exist a function $\eta: X \times X \rightarrow \mathbb{R}^{n}$ and a function $b: X \times X \rightarrow \mathbb{R}_{+} \backslash\{0\}$ such that, for all $x \in X$, the inequalities

$$
\begin{array}{r}
\frac{1}{r} b(x, u)\left(e^{r(f(x)-f(u))}-1\right) \geq \begin{cases}\frac{1}{p} \nabla f(u)\left(e^{p \eta(x, u)}-1\right) & (>\text { if } x \neq u) \text { for } p \neq 0, r \neq 0, \\
\nabla f(u) \eta(x, u) & (>\text { if } x \neq u) \text { for } p=0, r \neq 0,\end{cases} \\
b(x, u)(f(x)-f(u)) \geq \begin{cases}\frac{1}{p} \nabla f(u)\left(e^{p \eta(x, u)}-1\right) & (>\text { if } x \neq u) \text { for } p \neq 0, r=0, \\
\nabla f(u) \eta(x, u) & (>\text { if } x \neq u) \text { for } p=0, r=0\end{cases}
\end{array}
$$

hold.

$f$ is said to be $B$ - $(p, r)$-invex (strictly $B$ - $(p, r)$-invex) with respect to $\eta$ and $b$ on $X$ if it is $B$ - $(p, r)$-invex with respect to the same $\eta$ and $b$ at each $u \in X$.

It should be pointed out that exponentials appearing on the right-hand sides of the above inequalities are understood to be taken componentwise and $\mathbf{1}=(1,1, \ldots, 1) \in \mathbb{R}^{n}$. 

lem:

We consider the following nondifferentiable minimax fractional programming prob-

$$
\begin{aligned}
& \text { minimize } F(x)=\sup _{y \in Y} \frac{f(x, y)+\left(x^{\top} B x\right)^{1 / 2}}{h(x, y)-\left(x^{\top} D x\right)^{1 / 2}} \\
& \text { subject to } g(x) \leq 0,
\end{aligned}
$$

where $Y$ is a compact subset of $\mathbb{R}^{m}, f(\cdot, \cdot): \mathbb{R}^{n} \times \mathbb{R}^{m} \mapsto \mathbb{R}, h(\cdot, \cdot): \mathbb{R}^{n} \times \mathbb{R}^{m} \mapsto \mathbb{R}$, and $g(\cdot): \mathbb{R}^{n} \mapsto$ $\mathbb{R}^{p}$ are $C^{1}$-functions, $B$ and $D$ are $n \times n$ positive semidefinite matrices, $f(x, y)+\left(x^{\top} B x\right)^{1 / 2} \geq 0$, and $h(x, y)-\left(x^{\top} D x\right)^{1 / 2}>0$ for each $(x, y)$ in $X^{\circ} \times Y$, where $X^{\circ}$ is the set of feasible solutions of problem $(P)$; that is, $X^{\circ}=\{x \in X: g(x) \leq 0\}$. This is a nondifferentiable programming problem if either $B$ or $D$ is nonzero. If $B$ and $D$ are null matrices, then problem $(P)$ is a minimax fractional programming problem.

For each $(x, y) \in \mathbb{R}^{n} \times \mathbb{R}^{m}$ define

$$
\phi(x, y)=\frac{f(x, y)+\left(x^{\top} B x\right)^{1 / 2}}{h(x, y)-\left(x^{\top} D x\right)^{1 / 2}}
$$

We let

$$
\begin{aligned}
J= & \{1,2, \ldots, p\}, \\
J(x)=\left\{j \in J \mid g_{j}(x)=0\right\} & \\
Y(x)= & \left\{y \in Y \mid \phi(x, y)=\sup _{z \in Y} \phi(x, z)\right\}, \\
K(x)= & \left\{(s, t, y) \in \mathbb{N} \times \mathbb{R}_{+}^{s} \times \mathbb{R}^{m s} \mid 1 \leq s \leq n+1, t=\left(t_{1}, t_{2}, \ldots, t_{s}\right) \in \mathbb{R}_{+}^{s} \text { with } \sum_{i=1}^{s} t_{i}=1,\right. \\
& \left.y=\left(y_{1}, y_{2}, \ldots, y_{s}\right) \text { with } y_{i} \in Y(x), i=1,2, \ldots, s\right\} .
\end{aligned}
$$

Because $f$ and $h$ are contionuous differentiable and $Y$ is compact subset of $\mathbb{R}^{m}$, we see that for each $x_{0} \in X^{\circ}, Y\left(x_{0}\right) \neq \emptyset$, and for any $\bar{y}_{i} \in Y\left(x_{0}\right)$, we have a postive constant

$$
k_{0}=\phi\left(x_{0}, \bar{y}_{i}\right)=\frac{f\left(x_{0}, \bar{y}_{i}\right)+\left(x_{0}^{\top} B x_{0}\right)^{1 / 2}}{h\left(x_{0}, \bar{y}_{i}\right)-\left(x_{0}^{\top} D x_{0}\right)^{1 / 2}} .
$$

We will use the generalized Schwarz inequality

$$
x^{\top} B v \leq\left(x^{\top} B x\right)^{1 / 2}\left(v^{\top} B v\right)^{1 / 2}, \quad \text { for } x, v \in \mathbb{R}^{n} ;
$$

the equality holds when $B x=\lambda B v$, for some $\lambda \geq 0$. 
Hence if $v^{\top} B v \leq 1$, we have

$$
x^{\top} B v \leq\left(x^{\top} B x\right)^{1 / 2} .
$$

In [10] Lai et al. derived the following necessary conditions for optimality $(P)$.

Theorem 2.2 ((necessary conditions) see, [10]). Let $x_{0}$ be a $(P)$-optimal solution and satisfying $x_{0}^{\top} B x_{0}>0, x_{0}^{\top} D x_{0}>0$, and $\nabla g_{j}\left(x_{0}\right), j \in J\left(x_{0}\right)$ is linearly independent. Then there exist $\left(s, t^{*}, \bar{y}\right) \in$ $K\left(x_{0}\right), k_{0} \in \mathbb{R}_{+}, w, v \in \mathbb{R}^{n}$, and $\mu^{*} \in \mathbb{R}_{+}^{p}$ such that

$$
\begin{gathered}
\sum_{i=1}^{s} t_{i}^{*}\left\{\nabla f\left(x_{0}, \bar{y}_{i}\right)+B w-k_{0}\left(\nabla h\left(x_{0}, \bar{y}_{i}\right)-D v\right)\right\}+\nabla \sum_{j=1}^{p} \mu_{j}^{*} g_{j}\left(x_{0}\right)=0, \\
f\left(x_{0}, \bar{y}_{i}\right)+\left(x_{0}^{\top} B x_{0}\right)^{1 / 2}-k_{0}\left(h\left(x_{0}, \bar{y}_{i}\right)-\left(x_{0}^{\top} D x_{0}\right)^{1 / 2}\right)=0, \quad i=1,2, \ldots, s, \\
\sum_{j=1}^{p} \mu_{j}^{*} g_{j}\left(x_{0}\right)=0, \\
t_{i}^{*} \geq 0, \quad \sum_{i=1}^{s} t_{i}^{*}=1, \\
w^{\top} B w \leq 1, \quad v^{\top} D v \leq 1, \\
x_{0}^{\top} B w=\left(x_{0}^{\top} B x_{0}\right)^{1 / 2}, \\
x_{0}^{\top} D v=\left(x_{0}^{\top} B x_{0}\right)^{1 / 2} .
\end{gathered}
$$

It should be noted that both the matrices $B$ and $D$ are positive definite at the solution $x_{0}$ in the above theorem. If one of $x_{0}^{\top} B x_{0}$ and $x_{0}^{\top} D x_{0}$ is zero, or both $B$ and $D$ are singular at $x_{0}$, then, for $\left(s, t^{*}, \bar{y}\right) \in K\left(x_{0}\right)$, we define a set $Z_{\bar{y}}\left(x_{0}\right)$ by

$$
\begin{aligned}
& Z_{\bar{y}}\left(x_{0}\right) \\
& =\left\{z \in \mathbb{R}^{n} \mid z^{\top} \nabla g_{j}\left(x_{0}\right) \leq 0, j \in J\left(x_{0}\right) \text {, with any one of the next conditions (i)-(iii) holds }\right\} .
\end{aligned}
$$

Here conditions (i)-(iii) are given as follows:

(i) if $x_{0}^{\top} B x_{0}>0$ and $x_{0}^{\top} D x_{0}=0$, then

$$
z^{\top}\left(\sum_{i=1}^{s} t_{i}^{*}\left(\nabla f\left(x_{0}, \bar{y}_{i}\right)+\frac{B x_{0}}{\left(x_{0}^{\top} B x_{0}\right)^{1 / 2}}-k_{0} \nabla h\left(x_{0}, \bar{y}_{i}\right)\right)\right)+\left(z^{\top}\left(k_{0}^{2} D\right) z\right)^{1 / 2}<0
$$


(ii) if $x_{0}^{\top} B x_{0}=0$ and $x_{0}^{\top} D x_{0}>0$, then

$$
z^{\top}\left(\sum_{i=1}^{s} t_{i}^{*}\left(\nabla f\left(x_{0}, \bar{y}_{i}\right)-k_{0}\left(\nabla h\left(x_{0}, \bar{y}_{i}\right)-\frac{D x_{0}}{\left(x_{0}^{\top} D x_{0}\right)^{1 / 2}}\right)\right)\right)+\left(z^{\top} B z\right)^{1 / 2}<0,
$$

(iii) if $x_{0}^{\top} B x_{0}=0$ and $x_{0}^{\top} D x_{0}=0$, then

$$
z^{\top}\left(\sum_{i=1}^{s} t_{i}^{*}\left(\nabla f\left(x_{0}, \bar{y}_{i}\right)-k_{0} \nabla h\left(x_{0}, \bar{y}_{i}\right)\right)\right)+\left(z^{\top}\left(k_{0} D\right) z\right)^{1 / 2}+\left(z^{\top} B z\right)^{1 / 2}<0 .
$$
holds.

If we take condition $Z_{\bar{y}}\left(x_{0}\right)=\emptyset$ in Theorem 2.2, then the result of Theorem 2.2 still

\section{Optimality Conditions}

In this section we derive sufficient conditions for optimality of $(P)$ under the assumpition of a particular form of generalized $B-(p, r)$-invexity. All theorems in this work will be proved only in the case when $p \neq 0, r \neq 0$ (other cases can be dealt with by similarity since the only difference is arised from the form of the inequality defining the class of the $B$ - $(p, r)$-invex functions with respect to $\eta$ and $b$ for given $p$ and $r$ ). The proofs of the other cases are easier than this one.

We would establish the sufficient conditions under the $B$ - $(p, r)$-invex function.

Theorem 3.1 (sufficient optimality conditions). Let $x_{0} \in X^{\circ}$ be a feasible solution of $(P)$. There exist a positive interger $s, 1 \leq s \leq n+1, t^{*} \in \mathbb{R}^{s}, \bar{y}_{i} \in Y\left(x_{0}\right)(i=1,2, \ldots, s), k_{0} \in \mathbb{R}_{+},(w, v) \in$ $\mathbb{R}^{n} \times \mathbb{R}^{n}$, and $\mu^{*} \in \mathbb{R}_{+}^{p}$ to satisfy relations (2.7) (2.11). Furthermore suppose that any one of conditions (a) and (b) holds:

(a) $\Upsilon(\cdot)=\sum_{i=1}^{S} t_{i}^{*}\left(\left(f\left(\cdot, \bar{y}_{i}\right)+(\cdot)^{\top} B w\right)-k_{0}\left(h\left(\cdot, \bar{y}_{i}\right)-(\cdot)^{\top} D v\right)\right)$ is B-(p,r)-invex with respect to $\eta$ and $b$ at $x_{0}$, and $\sum_{j=1}^{p} \mu_{j}^{*} g_{j}(\cdot)$ is $B$ - $(p, r)$-invex with respect to the same function $\eta$ and another function $b_{1}$ at $x_{0}$ on $\mathrm{X}^{\circ}$, not necessarily, equal to $b$,

(b) $\Upsilon_{1}(\cdot)=\sum_{i=1}^{s} t_{i}^{*}\left(\left(f\left(\cdot, \bar{y}_{i}\right)+(\cdot)^{\top} B w\right)-k_{0}\left(h\left(\cdot, \bar{y}_{i}\right)-(\cdot)^{\top} D v\right)\right)+\sum_{j=1}^{p} \mu_{j}^{*} g_{j}(\cdot)$ is $B-(p, r)$-invex with respect to $\eta$ and $b$ at $x_{0}$ on $X^{\circ}$,

then $x_{0}$ is an optimal solution of $(P)$.

Proof. Suppose that $x_{0}$ is not an optimal solution of $(P)$. Then there exists a $(P)$-feasible solution $x_{1}$ such that

$$
\sup _{y \in Y} \frac{f\left(x_{1}, y\right)+\left(x_{1}^{\top} B x_{1}\right)^{1 / 2}}{h\left(x_{1}, y\right)-\left(x_{1}^{\top} D x_{1}\right)^{1 / 2}}<\sup _{y \in Y} \frac{f\left(x_{0}, y\right)+\left(x_{0}^{\top} B x_{0}\right)^{1 / 2}}{h\left(x_{0}, y\right)-\left(x_{0}^{\top} D x_{0}\right)^{1 / 2}} .
$$


We note that

$$
\sup _{y \in Y} \frac{f\left(x_{0}, y\right)+\left(x_{0}^{\top} B x_{0}\right)^{1 / 2}}{h\left(x_{0}, y\right)-\left(x_{0}^{\top} D x_{0}\right)^{1 / 2}}=\frac{f\left(x_{0}, \bar{y}_{i}\right)+\left(x_{0}^{\top} B x_{0}\right)^{1 / 2}}{h\left(x_{0}, \bar{y}_{i}\right)-\left(x_{0}^{\top} D x_{0}\right)^{1 / 2}}=k_{0},
$$

for $\bar{y}_{i} \in Y\left(x_{0}\right), i=1,2, \ldots, s$, and

$$
\frac{f\left(x_{1}, \bar{y}_{i}\right)+\left(x_{1}^{\top} B x_{1}\right)^{1 / 2}}{h\left(x_{1}, \bar{y}_{i}\right)-\left(x_{1}^{\top} D x_{1}\right)^{1 / 2}} \leq \sup _{y \in Y} \frac{f\left(x_{1}, y\right)+\left(x_{1}^{\top} B x_{1}\right)^{1 / 2}}{h\left(x_{1}, y\right)-\left(x_{1}^{\top} D x_{1}\right)^{1 / 2}}
$$

Then, we obtain

$$
\frac{f\left(x_{1}, \bar{y}_{i}\right)+\left(x_{1}^{\top} B x_{1}\right)^{1 / 2}}{h\left(x_{1}, \bar{y}_{i}\right)-\left(x_{1}^{\top} D x_{1}\right)^{1 / 2}}<k_{0}, \quad \text { for } i=1,2, \ldots, s
$$

It follows that

$$
f\left(x_{1}, \bar{y}_{i}\right)+\left(x_{1}^{\top} B x_{1}\right)^{1 / 2}-k_{0}\left(h\left(x_{1}, \bar{y}_{i}\right)-\left(x_{1}^{\top} D x_{1}\right)^{1 / 2}\right)<0, \quad \text { for } i=1,2, \ldots, s .
$$

From relations $(2.6),(2.11),(2.8),(2.10)$, and (3.5), we have

$$
\begin{aligned}
\Upsilon\left(x_{1}\right) & =\sum_{i=1}^{s} t_{i}^{*}\left(f\left(x_{1}, \bar{y}_{i}\right)+\left(x_{1}^{\top} B w\right)-k_{0}\left(h\left(x_{1}, \bar{y}_{i}\right)-\left(x_{1}^{\top} D v\right)\right)\right) \\
& \leq \sum_{i=1}^{s} t_{i}^{*}\left(f\left(x_{1}, \bar{y}_{i}\right)+\left(x_{1}^{\top} B x_{1}\right)^{1 / 2}-k_{0}\left(h\left(x_{1}, \bar{y}_{i}\right)-\left(x_{1}^{\top} D x_{1}\right)^{1 / 2}\right)\right)<0 \\
& =\sum_{i=1}^{s} t_{i}^{*}\left(f\left(x_{0}, \bar{y}_{i}\right)+\left(x_{0}^{\top} B x_{0}\right)^{1 / 2}-k_{0}\left(h\left(x_{0}, \bar{y}_{i}\right)-\left(x_{0}^{\top} D x_{0}\right)^{1 / 2}\right)\right) \\
& =\sum_{i=1}^{s} t_{i}^{*}\left(f\left(x_{0}, \bar{y}_{i}\right)+\left(x_{0}^{\top} B w\right)-k_{0}\left(h\left(x_{0}, \bar{y}_{i}\right)-\left(x_{0}^{\top} D v\right)\right)\right)=\Upsilon\left(x_{0}\right) .
\end{aligned}
$$

That is,

$$
\Upsilon\left(x_{1}\right)<\Upsilon\left(x_{0}\right)
$$

From relations $(P)$ and $(2.9)$, we obtain

$$
\sum_{j=1}^{p} \mu_{j}^{*} g_{j}\left(x_{1}\right) \leq 0=\sum_{j=1}^{p} \mu_{j}^{*} g_{j}\left(x_{0}\right)
$$


If hypothesis (a) holds, from the $B$ - $(p, r)$-invexity with respect to $\eta$ and $b_{1}$ at $x_{0}$ of $\sum_{j=1}^{p} \mu_{j}^{*} g_{j}(\cdot)$, we have

$$
\frac{1}{r} b_{1}\left(x_{1}, x_{0}\right)\left(e^{r\left(\sum_{j=1}^{p} \mu_{j}^{*} g_{j}\left(x_{1}\right)-\sum_{j=1}^{p} \mu_{j}^{*} g_{j}\left(x_{0}\right)\right)}-1\right) \geq \frac{1}{p} \nabla \sum_{j=1}^{p} \mu_{j}^{*} g_{j}\left(x_{0}\right)\left(e^{p \eta\left(x_{1}, x_{0}\right)}-\mathbf{1}\right) .
$$

From the inequalities (3.8) and (3.9), we get

$$
\frac{1}{p} \nabla\left(\sum_{j=1}^{p} \mu_{j}^{*} g_{j}\left(x_{0}\right)\right)\left(e^{p \eta\left(x_{1}, x_{0}\right)}-\mathbf{1}\right) \leq 0 .
$$

Now, multiplying equality $(2.7)$ by $(1 / p)\left(e^{p \eta\left(x_{1}, x_{0}\right)}-\mathbf{1}\right)$, we know

$$
\frac{1}{p}\left(e^{p \eta\left(x_{1}, x_{0}\right)}-\mathbf{1}\right)\left(\sum_{i=1}^{s} t_{i}^{*}\left\{\nabla f\left(x_{0}, \bar{y}_{i}\right)+B w-k_{0}\left(\nabla h\left(x_{0}, \bar{y}_{i}\right)-D v\right)\right\}+\nabla \sum_{j=1}^{p} \mu_{j}^{*} g_{j}\left(x_{0}\right)\right)=0 .
$$

From relations (3.10) and (3.11), we have

$$
\frac{1}{p}\left(e^{p \eta\left(x_{1}, x_{0}\right)}-\mathbf{1}\right)\left(\sum_{i=1}^{s} t_{i}^{*}\left\{\nabla f\left(x_{0}, \bar{y}_{i}\right)+B w-k_{0}\left(\nabla h\left(x_{0}, \bar{y}_{i}\right)-D v\right)\right\}\right) \geq 0 .
$$

From the $B$ - $(p, r)$-invexity with respect to the same function $\eta$ and the function $b$ at $x_{0}$ of $\Upsilon(\cdot)$,

$$
\frac{1}{r} b(x, u)\left(e^{r\left(\Upsilon\left(x_{1}\right)-\Upsilon\left(x_{0}\right)\right)}-1\right) \geq \frac{1}{p} \nabla \Upsilon\left(x_{0}\right)\left(e^{p \eta\left(x_{1}, x_{0}\right)}-\mathbf{1}\right) .
$$

From inequality (3.12) and the above inequality, we obtain

$$
\Upsilon\left(x_{1}\right) \geq \Upsilon\left(x_{0}\right),
$$

which contradicts (3.7), and proves that $x_{0}$ is an optimal solution to $(P)$. then

If hypothesis (b) holds, from the $B$ - $(p, r)$-invexity with respect to $\eta$ and $b$ at $x_{0}$ of $\Upsilon_{1}(\cdot)$,

$$
\frac{1}{r} b(x, u)\left(e^{r\left(\Upsilon_{1}\left(x_{1}\right)-\Upsilon_{1}\left(x_{0}\right)\right)}-1\right) \geq \frac{1}{p} \nabla \Upsilon_{1}\left(x_{0}\right)\left(e^{p \eta\left(x_{1}, x_{0}\right)}-\mathbf{1}\right) .
$$

The above inequality along with (2.7) yields

$$
\Upsilon_{1}\left(x_{1}\right) \geq \Upsilon_{1}\left(x_{0}\right)
$$

which contradicts (3.7). Hence, the proof is completed. 


\section{Parametric Dual-Type Model}

We use the optimality conditions of the preceding section and show that the following formation is a dual $(D)$ to the minimax problem $(P)$ :

$$
\max _{(s, t, y) \in K(z)} \sup _{(z, \mu, k, w, v) \in H_{1}(s, t, y)} k,
$$

where $H_{1}(s, t, y)$ denotes the set of $(z, \mu, k, w, v) \in \mathbb{R}^{n} \times \mathbb{R}_{+}^{p} \times \mathbb{R}_{+} \times \mathbb{R}^{n} \times \mathbb{R}^{n}$ satisfying

$$
\begin{gathered}
\sum_{i=1}^{s} t_{i}\left\{\nabla f\left(z, y_{i}\right)+B w-k\left(\nabla h\left(z, y_{i}\right)-D v\right)\right\}+\nabla \sum_{j=1}^{p} \mu_{j} g_{j}(z)=0 \\
\sum_{i=1}^{s} t_{i}\left\{f\left(z, \mathrm{y}_{i}\right)+z^{\top} B w-k\left(h\left(z, y_{i}\right)-z^{\top} D v\right)\right\} \geq 0 \\
\sum_{j=1}^{p} \mu_{j} g_{j}(z) \geq 0 \\
w^{\top} B w \leq 1, \quad v^{\top} D v \leq 1 \\
(s, t, y) \in K(z)
\end{gathered}
$$

If for a triplet $(s, t, y) \in K(z)$ the set $H_{1}(s, t, y)$ is empty, then we define the supremum over it to be $-\infty$.

Let $\Gamma$ denote the set of all feasible points of $(D)$. Moreover, we denote $\operatorname{pr}_{X} \Gamma=\{z \in X \mid$ $(z, \mu, s, t, y, w, v, k) \in \Gamma\}$.

We can derive the following weak duality theorem between $(P)$ and $(D)$.

Theorem 4.1 (weak duality). Let $x$ and $(z, \mu, s, t, y, w, v, k)$ be $(P)$-feasible and $(D)$-feasible, respectively. Suppose that any one of the following conditions $(a)$ and $(b)$ holds:

(a) $\Upsilon_{2}(\cdot)=\sum_{i=1}^{s} t_{i}\left(\left(f\left(\cdot, y_{i}\right)+(\cdot)^{\top} B w\right)-k\left(h\left(\cdot, y_{i}\right)-(\cdot)^{\top} D v\right)\right)$ is $B-(p, r)$-invex with respect to $\eta$ and $b$ at $z$ and $\sum_{j=1}^{p} \mu_{j} g_{j}(\cdot)$ is $B-(p, r)$-invex with respect to the same function $\eta$ and another function $b_{2}$ at $z$ on $X^{\circ} \cup p r_{X} \Gamma$, not necessarily, equal to $b$,

(b) $\Upsilon_{3}(\cdot)=\sum_{i=1}^{s} t_{i}\left(\left(f\left(\cdot, y_{i}\right)+(\cdot)^{\top} B w\right)-k\left(h\left(\cdot, y_{i}\right)-(\cdot)^{\top} D v\right)\right)+\sum_{j=1}^{p} \mu_{j} g_{j}(\cdot)$ is $(p, r)$-invex with respect to $\eta$ and $b$ at $z$ on $X^{\circ} \cup p r_{X} \Gamma$.

Then

$$
\sup _{y \in Y} \frac{f(x, y)+\left(x^{\top} B x\right)^{1 / 2}}{h(x, y)-\left(x^{\top} D x\right)^{1 / 2}} \geq k .
$$

Proof. Suppose on the contrary that

$$
\sup _{y \in Y} \frac{f(x, y)+\left(x^{\top} B x\right)^{1 / 2}}{h(x, y)-\left(x^{\top} D x\right)^{1 / 2}}<k .
$$


Then, we have an inequality

$$
f(x, y)+\left(x^{\top} B x\right)^{1 / 2}-k\left(h(x, y)-\left(x^{\top} D x\right)^{1 / 2}\right)<0 \quad \forall y \in Y .
$$

It follows that for $t_{i} \geq 0, i=1,2, \ldots, s$ with $\sum_{i=1}^{s} t_{i}=1$, we have

$$
t_{i}\left[f\left(x, y_{i}\right)+\left(x^{\top} B x\right)^{1 / 2}-k\left(h\left(x, y_{i}\right)-\left(x^{\top} D x\right)^{1 / 2}\right)\right] \leq 0,
$$

with at least one strict inequality because $t=\left(t_{1}, t_{2}, \ldots, t_{s}\right) \neq 0$. From relations (2.6), (4.4), (4.8), and (4.2), we obtain

$$
\begin{aligned}
\Upsilon_{2}(x) & =\sum_{i=1}^{s} t_{i}\left(f\left(x, y_{i}\right)+x^{\top} B w-k\left(h\left(x, y_{i}\right)-x^{\top} D v\right)\right) \\
& \leq \sum_{i=1}^{s} t_{i}\left[f\left(x, y_{i}\right)+\left(x^{\top} B x\right)^{1 / 2}-k\left(h\left(x, y_{i}\right)-\left(x^{\top} D x\right)^{1 / 2}\right)\right]<0 \\
& \leq \sum_{i=1}^{s} t_{i}\left(f\left(z, y_{i}\right)+z^{\top} B w-k\left(h\left(z, y_{i}\right)-z^{\top} D v\right)\right)=\Upsilon_{2}(z) .
\end{aligned}
$$

That is,

$$
\Upsilon_{2}(x)<\Upsilon_{2}(z) .
$$

From relations $(P)$ and (4.3), we have

$$
\sum_{j=1}^{p} \mu_{j} g_{j}(x) \leq 0 \leq \sum_{j=1}^{p} \mu_{j} g_{j}(z)
$$

If hypothesis (a) holds, from $B-(p, r)$-invexity with respect to $\eta$ and $b$ at $z$ of $\Upsilon_{2}(\cdot)$, we get

$$
\frac{1}{r} b(x, z)\left(\mathrm{e}^{r\left(\Upsilon_{2}(x)-\Upsilon_{2}(z)\right)}-1\right) \geq \frac{1}{p} \nabla \Upsilon_{2}(z)\left(e^{p \eta(x, z)}-\mathbf{1}\right) .
$$

From the above inequality together with relation (4.10), we have

$$
\frac{1}{p} \nabla \Upsilon_{2}(z)\left(e^{p \eta(x, z)}-1\right)<0
$$

Multiplying (4.1) by $(1 / p)\left(e^{p \eta(x, z)}-1\right)$, we obtain

$$
\frac{1}{p}\left(e^{p \eta(x, z)}-1\right)\left(\sum_{i=1}^{s} t_{i}\left\{\nabla f\left(z, y_{i}\right)+B w-k\left(\nabla h\left(z, y_{i}\right)-D v\right)\right\}+\nabla \sum_{j=1}^{p} \mu_{j} g_{j}(z)\right)=0 .
$$


From the above equality and inequality (4.13), we get

$$
\frac{1}{p}\left(e^{p \eta(x, z)}-\mathbf{1}\right) \sum_{j=1}^{p} \mu_{j} \nabla g_{j}(z)>0
$$

Using the $B-(p, r)$-invexity of $\sum_{j=1}^{p} \mu_{j} g_{j}(\cdot)$ with respect to the same function $\eta$ and the function $b_{2}$ at $z$ and inequality (4.15), we get

$$
\sum_{j=1}^{p} \mu_{j} g_{j}(x)>\sum_{j=1}^{p} \mu_{j} g_{j}(z)
$$

which contradicts (4.11) and proves that $\sup _{y \in Y}\left(\left(f(x, y)+\left(x^{\top} B x\right)^{1 / 2}\right) /\left(h(x, y)-\left(x^{\top} D x\right)^{1 / 2}\right)\right) \geq$ $k$. then

If hypothesis (b) holds, from the $B-(p, r)$-invexity with respect to $\eta$ and $b$ at $z$ of $\Upsilon_{3}(\cdot)$,

$$
\frac{1}{r} b(x, z)\left(e^{r\left(\Upsilon_{3}(x)-\Upsilon_{3}(z)\right)}-1\right) \geq \frac{1}{p} \nabla \Upsilon_{3}(z)\left(e^{p \eta(x, z)}-\mathbf{1}\right) .
$$

By the above inequality and equality (4.1), we have

$$
\Upsilon_{3}(x) \geq \Upsilon_{3}(z)
$$

From relations (4.10) and (4.11), we obtain

$$
\Upsilon_{3}(x)<\Upsilon_{3}(z),
$$

which contradicts inequality (4.18). Thus, the proof is complete.

Theorem 4.2 (strong duality). Let $x^{*}$ be an optimal solution of $(P)$, and let $x^{*}$ satisfy a constraint qualification for $(P)$. Then there exist $\left(s^{*}, t^{*}, y^{*}\right) \in K\left(x^{*}\right)$ and $\left(x^{*}, \mu^{*}, k^{*}, w^{*}, v^{*}\right) \in H_{1}\left(s^{*}, t^{*}, y^{*}\right)$ such that $\left(x^{*}, \mu^{*}, s^{*}, t^{*}, y^{*}, k^{*}, w^{*}, v^{*}\right)$ is a feasible solution of $(D)$. If in addition the hypothesis of Theorem 4.1 holds, then $\left(x^{*}, \mu^{*}, s^{*}, t^{*}, y^{*}, k^{*}, w^{*}, v^{*}\right)$ is an optimal solution of $(D)$ and the two problems $(P)$ and $(D)$ have the same optimal value.

Proof. By Theorem 2.2, there exist $\left(s^{*}, t^{*}, y^{*}\right) \in K\left(x^{*}\right)$ and $\left(x^{*}, \mu^{*}, k^{*}, w^{*}, v^{*}\right) \in H_{1}\left(s^{*}, t^{*}, y^{*}\right)$ such that $\left(x^{*}, \mu^{*}, s^{*}, t^{*}, y^{*}, k^{*}, w^{*}, v^{*}\right)$ is feasible for $(D)$, and

$$
k^{*}=\frac{f\left(x^{*}, y_{i}^{*}\right)+\left(x^{*^{\top}} B x^{*}\right)^{1 / 2}}{h\left(x^{*}, y_{i}^{*}\right)-\left(x^{*^{\top}} D x^{*}\right)^{1 / 2}} .
$$

The optimality of this feasible solution for $(D)$ follows from Theorem 4.1. 
Theorem 4.3 (strict converse duality). Let $\bar{x}$ and $\left(z^{*}, \mu^{*}, s^{*}, t^{*}, y^{*}, k^{*}, w^{*} v^{*}\right)$ be optimal solutions of $(P)$ and $(D)$, respectively, and assume that the hypothesis of Theorem 4.2 is fulfilled. Suppose that any one of the following conditions (a) and (b) holds:

(a) $\Upsilon_{4}(\cdot)=\sum_{i=1}^{s^{*}} t_{i}^{*}\left\{f\left(\cdot, y_{i}^{*}\right)+(\cdot)^{\top} B w^{*}-k^{*}\left(h\left(\cdot, y_{i}^{*}\right)-(\cdot)^{\top} D v^{*}\right)\right\}$ is strictly $B-(p, r)$-invex with respect to $\eta$ and $b$ at $z^{*}$ and $\sum_{j=1}^{p} \mu_{j}^{*} g_{j}(\cdot)$ is $(p, r)$-invex with respect to the same function $\eta$ and another function $b_{4}$ at $z^{*}$ on $X^{\circ} \cup p r_{X} \Gamma$, not necessarily, equal to $b$,

(b) $\Upsilon_{5}(\cdot)=\sum_{i=1}^{s^{*}} t_{i}^{*}\left\{f\left(\cdot, y_{i}^{*}\right)+(\cdot)^{\top} B w^{*}-k^{*}\left(h\left(\cdot, y_{i}^{*}\right)-(\cdot)^{\top} D v^{*}\right)\right\}+\sum_{j=1}^{p} \mu_{j}^{*} g_{j}(\cdot)$ is strictly $(p, r)$-invex with respect to $\eta$ and $b$ at $z^{*}$ on $X^{\circ} \cup p r_{X} \Gamma$.

Then $\bar{x}=z^{*}$, that is, $z^{*}$ solves $(P)$ and $\sup _{y \in Y}\left(\left(f\left(z^{*}, y\right)+\left(\left(z^{*}\right)^{\top} B z^{*}\right)^{1 / 2}\right) /\left(h\left(z^{*}, y\right)-\right.\right.$ $\left.\left.\left(\left(z^{*}\right)^{\top} D z^{*}\right)^{1 / 2}\right)\right)=k^{*}$.

Proof. We shall assume that $\bar{x} \neq z^{*}$ and reach a contradiction. From Theorem 4.2, we know that there exist $(\bar{s}, \bar{t}, \bar{y}) \in K(\bar{x})$ and $(\bar{x}, \bar{\mu}, \bar{k}, \bar{w}, \bar{v}) \in H_{1}(\bar{s}, \bar{t}, \bar{y})$ such that $(\bar{x}, \bar{\mu}, \bar{s}, \bar{t}, \bar{y}, \bar{k}, \bar{w}, \bar{v})$ is an optimal solution for $(D)$ with the optimal value

$$
\sup _{y \in Y} \frac{f(\bar{x}, y)+\left(\bar{x}^{\top} B \bar{x}\right)^{1 / 2}}{h(\bar{x}, y)-\left(\bar{x}^{\top} D \bar{x}\right)^{1 / 2}}=\bar{k}
$$

Now like the proof of Theorem 4.1 by $x$ replacing by $\bar{x}$ and $(z, \mu, s, t, y, w, v, k)$ by $\left(z^{*}, \mu^{*}, s^{*}, t^{*}, y^{*}, k^{*}, w^{*}, v^{*}\right)$, we obtain

$$
\sup _{y \in Y} \frac{f(\bar{x}, y)+\left(\bar{x}^{\top} B \bar{x}\right)^{1 / 2}}{h(\bar{x}, y)-\left(\bar{x}^{\top} D \bar{x}\right)^{1 / 2}}>k^{*}
$$

The above inequality contradicts

$$
\sup _{y \in \Upsilon} \frac{f(\bar{x}, y)+\left(\bar{x}^{\top} B \bar{x}\right)^{1 / 2}}{h(\bar{x}, y)-\left(\bar{x}^{\top} D \bar{x}\right)^{1 / 2}}=k^{*}=\bar{k}
$$

Therefore, we conclude that $\bar{x}=z^{*}$. Here, the proof of the theorem is complete.

Remark 4.4. In Theorem 4.3, if $\sum_{j=1}^{p} \mu_{j}^{*} g_{j}(\cdot)$ is a strictly $B$ - $(p, r)$-invex function with respect to $\eta$ and $b_{4}$ and $\Upsilon_{4}(\cdot)$ is a $B$ - $(p, r)$-invex function with respect to the same function $\eta$ and the function $b$, not necessarily, equal to $b_{4}$, then Theorem 4.3 also holds. 


\section{Mond-Weir Dual-Type Model}

In this section, we formulate the Mond-Weir-type dual model to the problem $(P)$ as follows:

$$
\max _{(s, t, y) \in K(z)} \sup _{(z, \mu, w, v) \in H_{2}(s, t, y)} \mathcal{F}(z)
$$

where $H_{2}(s, t, y)$ denotes the set of $(z, \mu, w, v) \in \mathbb{R}^{n} \times \mathbb{R}_{+}^{p} \times \mathbb{R}^{n} \times \mathbb{R}^{n}$ satisfying

$$
\begin{gathered}
\sum_{i=1}^{s} t_{i}\left\{\left(h\left(z, y_{i}\right)-\left(z^{\top} D z\right)^{1 / 2}\right)\left(\nabla f\left(z, y_{i}\right)+B w\right)\right. \\
\left.-\left(f\left(z, y_{i}\right)+\left(z^{\top} B z\right)^{1 / 2}\right)\left(\nabla h\left(z, y_{i}\right)-D v\right)\right\}+\sum_{j=1}^{p} \mu_{j} \nabla g_{j}(z)=0, \\
\sum_{j=1}^{p} \mu_{j} g_{j}(z) \geq 0, \\
w^{\top} B w \leq 1, \quad v^{\top} D v \leq 1, \\
z B w=\left(z^{\top} B z\right)^{1 / 2} \\
z D v=\left(z^{\top} B z\right)^{1 / 2},
\end{gathered}
$$

where

$$
\mathcal{F}(z)=\sup _{y \in Y} \frac{f(z, y)+\left(z^{\top} B z\right)^{1 / 2}}{h(z, y)-\left(z^{\top} D z\right)^{1 / 2}}=\sup _{y \in Y} \phi(z, y) .
$$

If for a triplet $(s, t, y) \in K(z)$ the set $H_{2}(s, t, y)$ is empty, then we define the supremum over it to be $-\infty$.

Let $\widetilde{\Gamma}$ denote the set of all feasible points of $(M W D)$. Moreover, we denote $p r_{X} \widetilde{\Gamma}=\{z \in$ $X \mid(z, \mu, s, t, y, w, v) \in \tilde{\Gamma}\}$.

We establish the weak, strong, and strict converse duality theorems for $(M W D)$ with respect to the primal problem $(P)$.

Theorem 5.1 (weak duality). Let $x$ and $(z, \mu, s, t, y, w, v)$ be $(P)$-feasible and (MWD)-feasible, respectively. Suppose that any one of the following conditions (a) and (b) holds:

(a) $\Upsilon_{6}(\cdot)=\sum_{i=1}^{s} t_{i}\left\{\left(h\left(z, y_{i}\right)-z^{\top} D v\right)\left(f\left(\cdot, y_{i}\right)+(\cdot)^{\top} B w\right)-\left(f\left(z, y_{i}\right)+z^{\top} B w\right)\left(h\left(\cdot, y_{i}\right)-\right.\right.$ $\left.\left.(\cdot)^{\top} D v\right)\right\}$ is $B-(p, r)$-invex, respect to $\eta$ and $b$ at $z$ and $\sum_{j=1}^{p} \mu_{j} g_{j}(\cdot)$ is $B-(p, r)$-invex with respect to the same function $\eta$ and another function $b_{6}$ at $z$ on $X^{\circ} \cup \widetilde{\Gamma}$, not necessarily, equal to $b$,

(b) $\Upsilon_{7}=\sum_{i=1}^{s} t_{i}\left\{\left(h\left(z, y_{i}\right)-z^{\top} D v\right)\left(f\left(\cdot, y_{i}\right)+(\cdot)^{\top} B w\right)-\left(f\left(z, y_{i}\right)+z^{\top} B w\right)\left(h\left(\cdot, y_{i}\right)-(\cdot)^{\top} D v\right)\right\}+$ $\sum_{j=1}^{p} \mu_{j} g_{j}(\cdot)$ is $B-(p, r)$-invex with respect to $\eta$ and $b$ at $z$ on $X^{\circ} \cup \widetilde{\Gamma}$. 
Then

$$
\sup _{y \in Y} \frac{f(x, y)+\left(x^{\top} B x\right)^{1 / 2}}{h(x, y)-\left(x^{\top} D x\right)^{1 / 2}} \geq \mathcal{F}(z)
$$

Proof. On the contrary, if possible, suppose that for each $x \in X^{\circ}$,

$$
\sup _{y \in Y} \frac{f(x, y)+\left(x^{\top} B x\right)^{1 / 2}}{h(x, y)-\left(x^{\top} D x\right)^{1 / 2}}<F(z)
$$

From the above inequality and $y_{i} \in Y(z), i=1,2, \ldots, s$, we obtain

$$
\frac{f\left(x, y_{i}\right)+\left(x^{\top} B x\right)^{1 / 2}}{h\left(x, y_{i}\right)-\left(x^{\top} D x\right)^{1 / 2}}<F(z)=\frac{f\left(z, y_{i}\right)+\left(z^{\top} B z\right)^{1 / 2}}{h\left(z, y_{i}\right)-\left(z^{\top} D z\right)^{1 / 2}}, \quad i=1,2, \ldots, s
$$

By the above inequality, we know that

$$
\begin{aligned}
& \left(f\left(x, y_{i}\right)+\left(x^{\top} B x\right)^{1 / 2}\right)\left(h\left(z, y_{i}\right)-\left(z^{\top} D z\right)^{1 / 2}\right) \\
& -\left(h\left(x, y_{i}\right)-\left(x^{\top} D x\right)^{1 / 2}\right)\left(f\left(z, y_{i}\right)+\left(z^{\top} B z\right)^{1 / 2}\right)<0
\end{aligned}
$$

for all $i=1,2, \ldots, s$ and $y_{i} \in Y(z)$.

Multiplying the above inequality by $t_{i} \in \mathbb{R}_{+}^{s}$ with $\sum_{i=1}^{s} t_{i}=1$, we have

$$
\begin{aligned}
\sum_{i=1}^{s} t_{i}\{( & \left.f\left(x, y_{i}\right)+\left(x^{\top} B x\right)^{1 / 2}\right)\left(h\left(z, y_{i}\right)-\left(z^{\top} D z\right)^{1 / 2}\right) \\
- & \left.\left(h\left(x, y_{i}\right)-\left(x^{\top} D x\right)^{1 / 2}\right)\left(f\left(z, y_{i}\right)+\left(z^{\top} B z\right)^{1 / 2}\right)\right\}<0 .
\end{aligned}
$$

From relations (2.6), (5.3), and (5.9), we get

$$
\begin{aligned}
& \Upsilon_{6}(x)= \sum_{i=1}^{s} t_{i}\left\{\left(f\left(x, y_{i}\right)+x^{\top} B w\right)\left(h\left(z, y_{i}\right)-z^{\top} D v\right)-\left(h\left(x, y_{i}\right)-x^{\top} D v\right)\left(f\left(z, y_{i}\right)+z^{\top} B w\right)\right\} \\
& \leq \sum_{i=1}^{s} t_{i}\left\{\left(f\left(x, y_{i}\right)+\left(x^{\top} B x\right)^{1 / 2}\right)\left(h\left(z, y_{i}\right)-\left(z^{\top} D z\right)^{1 / 2}\right)\right. \\
&\left.\quad-\left(h\left(x, y_{i}\right)-\left(x^{\top} D x\right)^{1 / 2}\right)\left(f\left(z, y_{i}\right)+\left(z^{\top} B z\right)^{1 / 2}\right)\right\} \\
&<0=\Upsilon_{6}(z) .
\end{aligned}
$$


By relations $(P)$ and (5.2), we have

$$
\sum_{j=1}^{p} \mu_{j} g_{j}(x) \leq 0 \leq \sum_{j=1}^{p} \mu_{j} g_{j}(z)
$$

Now, if condition (a) holds, from $B$ - $(p, r)$-invexity with respect to $\eta$ and $b_{6}$ at $z$ of $\sum_{j=1}^{p} \mu_{j} g_{j}(\cdot)$, we get

$$
\frac{1}{r} b_{1}\left(x_{1}, x_{0}\right)\left(e^{r\left(\sum_{j=1}^{p} \mu_{j}^{*} g_{j}(x)-\sum_{j=1}^{p} \mu_{j}^{*} g_{j}(z)\right)}-1\right) \geq \frac{1}{p} \nabla \sum_{j=1}^{p} \mu_{j}^{*} g_{j}(z)\left(e^{p \eta(x, z)}-\mathbf{1}\right) .
$$

From the above inequality and inequality (5.11), we obtain

$$
\frac{1}{p} \nabla \sum_{j=1}^{p}\left(\mu_{j} g_{j}(z)\right)\left(e^{p \eta(x, z)}-\mathbf{1}\right) \leq 0
$$

Multiplying (5.1) by $(1 / p)\left(e^{p \eta(x, z)}-1\right)$, we have

$$
\begin{aligned}
\frac{1}{p}\left(e^{p \eta(x, z)}-1\right)\left\{\sum_{i=1}^{s} t_{i}\{\right. & \left(h\left(z, y_{i}\right)-\left(z^{\top} D z\right)^{1 / 2}\right)\left(\nabla f\left(z, y_{i}\right)+B w\right) \\
& \left.\left.-\left(f\left(z, y_{i}\right)+\left(z^{\top} B z\right)^{1 / 2}\right)\left(\nabla h\left(z, y_{i}\right)-D v\right)\right\}+\sum_{j=1}^{p} \mu_{j} \nabla g_{j}(z)\right\}=0 .
\end{aligned}
$$

By the above equality and inequality (5.13), we obtain

$$
\begin{aligned}
\frac{1}{p}\left(e^{p \eta(x, z)}-1\right)\left\{\sum_{i=1}^{s} t_{i}\{\right. & \left(h\left(z, y_{i}\right)-\left(z^{\top} D z\right)^{1 / 2}\right)\left(\nabla f\left(z, y_{i}\right)+B w\right) \\
& \left.\left.-\left(f\left(z, y_{i}\right)+\left(z^{\top} B z\right)^{1 / 2}\right)\left(\nabla h\left(z, y_{i}\right)-D v\right)\right\}\right\} \geq 0 .
\end{aligned}
$$

Using the $B-(p, r)$-invexity with respect to the same function $\eta$ and the function $b$ at $z$ of $\Upsilon_{6}(\cdot)$ and the above inequality, we have

$$
\Upsilon_{6}(x) \geq \Upsilon_{6}(z)
$$

which contradicts (5.10) and proves that $\sup _{y \in Y}\left(\left(f(x, y)+\left(x^{\top} B x\right)^{1 / 2}\right) /\left(h(x, y)-\left(x^{\top} D x\right)^{1 / 2}\right)\right) \geq$ $f(z)$. 
If hypothesis (b) holds, from the $B$ - $(p, r)$-invexity with respect to $\eta$ and $b$ at $z$ of $\Upsilon_{7}(\cdot)$ and the equality (5.1), then

$$
\Upsilon_{7}(x) \geq \Upsilon_{7}(z)
$$

From relations (5.10) and (5.11), we have

$$
\begin{aligned}
& \sum_{i=1}^{s} t_{i}\left\{\left(h\left(z, y_{i}\right)-z^{\top} D v\right)\left(f\left(x, y_{i}\right)+x^{\top} B w\right)-\left(f\left(z, y_{i}\right)+z^{\top} B w\right)\left(h\left(x, y_{i}\right)-x^{\top} D v\right)\right\} \\
& \quad+\sum_{j=1}^{p} \mu_{j} g_{j}(x) \\
& \quad<\sum_{i=1}^{s} t_{i}\left\{\left(h\left(z, y_{i}\right)-z^{\top} D v\right)\left(f\left(z, y_{i}\right)+z^{\top} B w\right)-\left(f\left(z, y_{i}\right)+z^{\top} B w\right)\left(h\left(z, y_{i}\right)-z^{\top} D v\right)\right\} \\
& \quad+\sum_{j=1}^{p} \mu_{j} g_{j}(z),
\end{aligned}
$$

which contradicts inequality (5.17). Hence, the proof is complete.

Similar to the proof of Theorem 4.2, we can establish Theorem 5.2.

Theorem 5.2 (strong duality). Let $x^{*}$ be an optimal solution of $(P)$ and let $x^{*}$ satisfy a constraint qualification for $(P)$. Then there exist $\left(s^{*}, t^{*}, y^{*}\right) \in K\left(x^{*}\right)$ and $\left(x^{*}, \mu^{*}, w^{*}, v^{*}\right) \in H_{1}\left(s^{*}, t^{*}, y^{*}\right)$ such that $\left(x^{*}, \mu^{*}, s^{*}, t^{*}, y^{*}, w^{*}, v^{*}\right)$ is a feasible solution of (MWD). If in addition the hypothesis of Theorem 5.1 holds, then $\left(x^{*}, \mu^{*}, s^{*}, t^{*}, y^{*}, w^{*}, v^{*}\right)$ is an optimal solution of $(M W D)$ and the two problems $(P)$ and $(M W D)$ have the same optimal value.

Theorem 5.3 (strict converse duality). Let $\bar{x}$ and $\left(z^{*}, \mu^{*}, s^{*}, t^{*}, y^{*}, w^{*}, v^{*}\right)$ be optimal solutions of $(P)$ and $(M W D)$, respectively, and assume that the hypothesis of Theorem 5.2 is fulfilled. Suppose that any one of the following conditions (a) and (b) holds:

(a) $\Upsilon_{8}(\cdot)=\sum_{i=1}^{s^{*}} t_{i}^{*}\left\{\left(h\left(z^{*}, y_{i}^{*}\right)-\left(z^{*}\right)^{\top} D v\right)\left(f\left(\cdot, y_{i}^{*}\right)+(\cdot)^{\top} B w\right)-\left(f\left(z^{*}, y_{i}^{*}\right)+\left(z^{*}\right)^{\top} B w\right)(h(\cdot\right.$, $\left.\left.\left.y_{i}^{*}\right)-(\cdot)^{\top} D v\right)\right\}$ is strictly $B-(p, r)$-invex with respect to $\eta$ and $b$ at $z^{*}$ and $\sum_{j=1}^{p} \mu_{j}^{*} g_{j}(\cdot)$ is $B-(p, r)$-invex with respect to the same function $\eta$ and another function $b_{8}$ at $z^{*}$ on $X^{\circ} \cup \tilde{\Gamma}$, not necessarily, equal to $b$,

(b) $\Upsilon_{9}(\cdot)=\sum_{i=1}^{s^{*}} t_{i}^{*}\left\{\left(h\left(z^{*}, y_{i}^{*}\right)-\left(z^{*}\right)^{\top} D v\right)\left(f\left(\cdot, y_{i}^{*}\right)+(\cdot)^{\top} B w\right)-\left(f\left(z^{*}, y_{i}^{*}\right)+\left(z^{*}\right)^{\top} B w\right)(h(\cdot\right.$, $\left.\left.\left.y_{i}^{*}\right)-(\cdot)^{\top} D v\right)\right\}+\sum_{j=1}^{p} \mu_{j}^{*} g_{j}(\cdot)$ is strictly $B-(p, r)$-invex with respect to $\eta$ and $b$ at $z^{*}$ on $X^{\circ} \cup \tilde{\Gamma}$.

Then $\bar{x}=z^{*}$, that is, $z^{*}$ solves $(P)$ and $\sup _{y \in Y}\left(\left(f\left(z^{*}, y\right)+\left(\left(z^{*}\right)^{\top} B z^{*}\right)^{1 / 2}\right) /\left(h\left(z^{*}, y\right)-\right.\right.$ $\left.\left.\left(\left(z^{*}\right)^{\top} D z^{*}\right)^{1 / 2}\right)\right)=\mathcal{F}\left(z^{*}\right)$. 
Proof. We suppose on the contrary that if $\bar{x} \neq z^{*}$, then

$$
\sup _{y \in Y} \frac{f(\bar{x}, y)+\left(\bar{x}^{\top} B \bar{x}\right)^{1 / 2}}{h(\bar{x}, y)-\left(\bar{x}^{\top} D \bar{x}\right)^{1 / 2}} \leq \mathcal{F}\left(z^{*}\right)
$$

Since $y_{i}^{*} \in Y\left(z^{*}\right)$, for $i=1,2, \ldots, s^{*}$,

$$
\frac{f\left(z^{*}, y_{i}^{*}\right)+\left(\left(z^{*}\right)^{\top} B z^{*}\right)^{1 / 2}}{h\left(z^{*}, y_{i}^{*}\right)-\left(\left(z^{*}\right)^{\top} D z^{*}\right)^{1 / 2}}=\mathcal{F}\left(z^{*}\right), \text { for } i=1,2, \ldots, s^{*}
$$

It follows that

$$
\begin{gathered}
\left(f(\bar{x}, y)+\left(\bar{x}^{\top} B \bar{x}\right)^{1 / 2}\right)\left(h\left(z^{*}, y_{i}^{*}\right)-\left(\left(z^{*}\right)^{\top} D z^{*}\right)^{1 / 2}\right) \\
-\left(h(\bar{x}, y)-\left(\bar{x}^{\top} D \bar{x}\right)^{1 / 2}\right)\left(f\left(z^{*}, y_{i}^{*}\right)+\left(z^{*}\right)^{\top} B z^{*}\right)^{1 / 2}
\end{gathered}
$$

$\leq 0, \quad$ for $i=1,2, \ldots, s^{*}, y \in Y$.

From $y_{i}^{*} \in Y\left(z^{*}\right) \subset Y$ and $t \in \mathbb{R}_{+}^{s^{*}}$ with $\sum_{i=1}^{s^{*}} t_{i}^{*}=1$, we have

$$
\begin{aligned}
\sum_{i=1}^{s^{*}} t_{i}^{*}\{ & \left(h\left(z^{*}, y_{i}^{*}\right)-\left(\left(z^{*}\right)^{\top} D z^{*}\right)^{1 / 2}\right)\left(f\left(\bar{x}, y_{i}^{*}\right)+\left(\bar{x}^{\top} B \bar{x}\right)^{1 / 2}\right) \\
& \left.-\left(h\left(\bar{x}, y_{i}^{*}\right)-\left(\bar{x}^{\top} D \bar{x}\right)^{1 / 2}\right)\left(f\left(z^{*}, y_{i}^{*}\right)+\left(\left(z^{*}\right)^{\top} B z^{*}\right)^{1 / 2}\right)\right\} \leq 0 .
\end{aligned}
$$

From relations (2.6), (5.3), and inequality (5.22), we obtain that

$$
\begin{aligned}
& \Upsilon_{8}(\bar{x})=\sum_{i=1}^{s^{*}} t_{i}^{*}\{(\left.h\left(z^{*}, y_{i}^{*}\right)-\left(z^{*}\right)^{\top} D v\right)\left(f\left(\bar{x}, y_{i}^{*}\right)+\bar{x}^{\top} B w\right) \\
&\left.-\left(f\left(z^{*}, y_{i}^{*}\right)+\left(z^{*}\right)^{\top} B w\right)\left(h\left(\bar{x}, y_{i}^{*}\right)-\bar{x}^{\top} D v\right)\right\} \\
& \leq \sum_{i=1}^{s^{*}} t_{i}^{*}\left\{\left(h\left(z^{*}, y_{i}^{*}\right)-\left(\bar{x}^{\top} D \bar{x}\right)^{1 / 2}\right)\left(f\left(z^{*}, y_{i}^{*}\right)+\left(\left(z^{*}\right)^{\top} B z^{*}\right)^{1 / 2}\right)\right.\left.-\left(h\left(\bar{x}, y_{i}^{*}\right)-\left(\bar{x}^{\top} D \bar{x}\right)^{1 / 2}\right)\left(f\left(z^{*}, y_{i}^{*}\right)+\left(\left(z^{*}\right)^{\top} B z^{*}\right)^{1 / 2}\right)\right\} \\
& \leq 0=\Upsilon_{8}\left(z^{*}\right) .
\end{aligned}
$$


By relations $(P)$ and (5.2), we get

$$
\sum_{j=1}^{p} \mu_{j}^{*} g_{j}(\bar{x}) \leq 0 \leq \sum_{j=1}^{p} \mu_{j}^{*} g_{j}\left(z^{*}\right)
$$

Now, if condition (a) holds, from $B$ - $(p, r)$-invexity with respect to $\eta$ and $b_{8}$ at $z$ of $\sum_{j=1}^{p} \mu_{j}^{*} g_{j}(\cdot)$, then

$$
\frac{1}{r} b_{1}\left(\bar{x}, z^{*}\right)\left(e^{r\left(\sum_{j=1}^{p} \mu_{j}^{*} g_{j}(\bar{x})-\sum_{j=1}^{p} \mu_{j}^{*} g_{j}\left(z^{*}\right)\right)}-1\right) \geq \frac{1}{p} \nabla \sum_{j=1}^{p} \mu_{j}^{*} g_{j}\left(z^{*}\right)\left(e^{p \eta\left(\bar{x}, z^{*}\right)}-\mathbf{1}\right) .
$$

From the above inequality and inequality (5.24), we know that

$$
\frac{1}{p} \nabla \sum_{j=1}^{p} \mu_{j}^{*} g_{j}\left(z^{*}\right)\left(e^{p \eta\left(\bar{x}, z^{*}\right)}-\mathbf{1}\right) \leq 0
$$

Multiplying $(M W D)$ by $(1 / p)\left(e^{p \eta\left(\bar{x}, z^{*}\right)}-1\right)$, we have

$$
\begin{gathered}
\frac{1}{p}\left(e^{p \eta\left(\bar{x}, z^{*}\right)}-1\right)\left\{\sum _ { i = 1 } ^ { s ^ { * } } t _ { i } ^ { * } \left\{\left(h\left(z^{*}, y_{i}^{*}\right)-\left(\left(z^{*}\right)^{\top} D z^{*}\right)^{1 / 2}\right)\left(\nabla f\left(z^{*}, y_{i}^{*}\right)+B w^{*}\right)\right.\right. \\
\left.\quad-\left(f\left(z^{*}, y_{i}^{*}\right)+\left(\left(z^{*}\right)^{\top} B z^{*}\right)^{1 / 2}\right)\left(\nabla h\left(z^{*}, y_{i}^{*}\right)-D v\right)\right\} \\
\left.+\sum_{j=1}^{p} \mu_{j}^{*} \nabla g_{j}\left(z^{*}\right)\right\}=0 .
\end{gathered}
$$

Basing on the above inequality and (5.26), we get the inequality

$$
\begin{aligned}
\frac{1}{p}\left(e^{p \eta\left(\bar{x}, z^{*}\right)}-1\right)\left\{\sum_{i=1}^{s^{*}} t_{i}^{*}\{(\right. & \left.h\left(z^{*}, y_{i}^{*}\right)-\left(\left(z^{*}\right)^{\top} D z^{*}\right)^{1 / 2}\right)\left(\nabla f\left(z^{*}, y_{i}^{*}\right)+B w^{*}\right) \\
& \left.\left.-\left(f\left(z^{*}, y_{i}^{*}\right)+\left(\left(z^{*}\right)^{\top} B z^{*}\right)^{1 / 2}\right)\left(\nabla h\left(z^{*}, y_{i}^{*}\right)-D v\right)\right\}\right\} \geq 0 .
\end{aligned}
$$

Since $\Upsilon_{8}(\cdot)$ is strictly $B$ - $(p, r)$-invex with respect to the same function $\eta$ and the function $b$ at $z^{*}$ and the above inequality, we obtain

$$
\Upsilon_{8}\left(z^{*}\right)<\Upsilon_{8}(\bar{x})
$$

which contradicts relations (5.23). Hence $\bar{x}=z^{*}$ and $z^{*}$ is an optimal solution of $(P)$. 
If hypothesis (b) holds, from the strict $B-(p, r)$-invexity with respect to $\eta$ and $b$ at $z^{*}$ of $\Upsilon_{9}(\cdot)$ and equality $(5.1)$, then

$$
\Upsilon_{9}(\bar{x})>\Upsilon_{9}\left(z^{*}\right)
$$

Relations (5.23) together with (5.24) yield

$$
\begin{aligned}
& \sum_{i=1}^{s^{*}} t_{i}^{*}\left\{\left(h\left(z^{*}, y_{i}^{*}\right)-\left(z^{*}\right)^{\top} D v\right)\left(f\left(\bar{x}, y_{i}^{*}\right)+\bar{x}^{\top} B w\right)\right. \\
& \left.-\left(f\left(z^{*}, y_{i}^{*}\right)+z^{\top} B w\right)\left(h\left(\bar{x}, y_{i}^{*}\right)-\bar{x}^{\top} D v\right)\right\}+\sum_{j=1}^{p} \mu_{j}^{*} g_{j}(\bar{x}) \\
& \leq \sum_{i=1}^{s^{*}} t_{i}^{*}\left\{\left(h\left(z^{*}, y_{i}^{*}\right)-\left(z^{*}\right)^{\top} D v\right)\left(f\left(z^{*}, y_{i}^{*}\right)+\left(z^{*}\right)^{\top} B w\right)\right. \\
& \left.-\left(f\left(z^{*}, y_{i}^{*}\right)+\left(z^{*}\right)^{\top} B w\right)\left(h\left(z^{*}, y_{i}^{*}\right)-\left(z^{*}\right)^{\top} D v\right)\right\}+\sum_{j=1}^{p} \mu_{j}^{*} g_{j}\left(z^{*}\right)
\end{aligned}
$$

which contradicts (5.30). Hence $z^{*}=\bar{x}$ is also an optimal solution of $(P)$. The proof is complete.

Remark 5.4. In Theorem 5.3, if $\sum_{j=1}^{p} \mu_{j}^{*} g_{j}(\cdot)$ is a strictly $B$ - $(p, r)$-invex function with respect to $\eta$ and $b_{8}$ and $\Upsilon_{8}(\cdot)$ is a $B-(p, r)$-invex function with respect to the same function $\eta$ and the function $b$, not necessarily, equal to $b_{8}$, then Theorem 5.3 also holds.

\section{Wolfe Dual-Type Model}

Based on the result of Theorem 2.2, we can rewrite Theorem 2.2 as follows.

Theorem 6.1 ((necessary conditions) Lai and Lee [11, Theorem 4]). Let $x_{0}$ be a $(P)$-optimal solution, and let $\nabla g_{j}\left(x_{0}\right), j \in J\left(x_{0}\right)$ be linearly independent. Then there exist $\left(s^{*}, t^{*}, y^{*}\right) \in$ $K\left(x_{0}\right),(w, v) \in \mathbb{R}^{n} \times \mathbb{R}^{n}$, and $\mu^{*} \in \mathbb{R}_{+}^{p}$ such that

$$
\begin{gathered}
\nabla\left(\frac{\sum_{i=1}^{s^{*}} t_{i}^{*} f\left(x_{0}, y_{i}^{*}\right)+x_{0}^{\top} B w+\sum_{j=1}^{p} \mu_{j}^{*} g_{j}\left(x_{0}\right)}{\sum_{i=1}^{s^{*}} t_{i}^{*}\left(h\left(x_{0}, y_{i}^{*}\right)-x_{0}^{\top} D v\right)}\right)=0, \\
\sum_{j=1}^{p} \mu_{j}^{*} g_{j}\left(x_{0}\right)=0, \\
t_{i}^{*} \geq 0, \sum_{i=1}^{s^{*}} t_{i}^{*}=1, \\
w^{\top} B w \leq 1, \quad v^{\top} D v \leq 1, \\
x_{0}^{\top} B w=\left(x_{0}^{\top} B x_{0}\right)^{1 / 2}, \\
x_{0}^{\top} D v=\left(x_{0}^{\top} B x_{0}\right)^{1 / 2} .
\end{gathered}
$$


In this section, we present the Wolfe dual $(W D)$ to the minimax program $(P)$ :

$$
\max _{(s, t, y) \in K(z)} \sup _{(z, \mu, w, v) \in H_{3}(s, t, y)} \mathcal{A}(z)
$$

where $H_{3}(s, t, y)$ denotes the set of $(z, \mu, w, v) \in \mathbb{R}^{n} \times \mathbb{R}_{+}^{p} \times \mathbb{R}^{n} \times \mathbb{R}^{n}$ satisfying

$$
\begin{gathered}
\nabla\left(\frac{\sum_{i=1}^{s} t_{i}\left(f\left(z, y_{i}\right)+z^{\top} B w\right)+\sum_{j=1}^{p} \mu_{j} g_{j}(z)}{\sum_{i=1}^{s} t_{i}\left(h\left(z, y_{i}\right)-z^{\top} D v\right)}\right)=0, \quad(s, t, y) \in K, \\
w^{\top} B w \leq 1, \quad v^{\top} D v \leq 1, \\
z^{\top} B w=\left(z^{\top} B z\right)^{1 / 2}, \\
z^{\top} D v=\left(z^{\top} B z\right)^{1 / 2}, \\
A(z)=\frac{\sum_{i=1}^{s} t_{i}\left(f\left(z, y_{i}\right)+z^{\top} B w\right)+\sum_{j=1}^{p} \mu_{j} g_{j}(z)}{\sum_{i=1}^{s} t_{i}\left(h\left(z, y_{i}\right)-z^{\top} D v\right)} .
\end{gathered}
$$

If for a triplet $(s, t, y) \in K(z)$ the set $H_{3}(s, t, y)$ is empty, then we define the supremum over it to be $-\infty$.

Let $\bar{\Gamma}$ denote the set of all feasible points of $(W D)$. Moreover, we denote $p r_{X} \bar{\Gamma}=\{z \in$ $X \mid(z, \mu, s, t, y, w, v) \in \bar{\Gamma}\}$.

We assume throughout this section that $\sum_{i=1}^{s} t_{i}\left(f\left(z, y_{i}\right)+z^{\top} B w\right)+\sum_{j=1}^{p} \mu_{j} g_{j}(z) \geq 0$ and $\sum_{i=1}^{s} t_{i}\left(h\left(z, y_{i}\right)-z^{\top} D v\right)>0$.

We establish the weak, strong, and strict converse duality theorems for $(W D)$ with respect to the primal problem $(P)$.

Theorem 6.2 (weak duality). Let $x$ and $(z, \mu, s, t, y, w, v)$ be (P)-feasible and (WD)-feasible, respectively, and assume that

$$
\begin{aligned}
\Upsilon_{10}(\cdot)= & {\left[\sum_{i=1}^{s} t_{i}\left(h\left(z, y_{i}\right)-z^{\top} D v\right)\right]\left[\sum_{i=1}^{s} t_{i}\left(f\left(\cdot, y_{i}\right)+(\cdot)^{\top} B w\right)+\sum_{j=1}^{p} \mu_{j} g_{j}(\cdot)\right] } \\
& -\left[\sum_{i=1}^{s} t_{i}\left(f\left(z, y_{i}\right)+z^{\top} B w\right)+\sum_{j=1}^{p} \mu_{j} g_{j}(z)\right]\left[\sum_{i=1}^{s} t_{i}\left(h\left(\cdot, y_{i}\right)-(\cdot)^{\top} D v\right)\right]
\end{aligned}
$$

is $B$ - $(p, r)$-invex with respect to $\eta$ and $b$ at $z$ on $X^{\circ} \cup p r_{X} \bar{\Gamma}$; then

$$
\sup _{y \in Y} \frac{f(x, y)+\left(x^{\top} B x\right)^{1 / 2}}{h(x, y)-\left(x^{\top} D x\right)^{1 / 2}} \geq \mathcal{A}(z)
$$


Proof. Suppose on the contrary that

$$
\sup _{y \in Y} \frac{f(x, y)+\left(x^{\top} B x\right)^{1 / 2}}{h(x, y)-\left(x^{\top} D x\right)^{1 / 2}}<A(z) .
$$

Hence, we have an inequality

$$
\frac{f(x, y)+\left(x^{\top} B x\right)^{1 / 2}}{h(x, y)-\left(x^{\top} D x\right)^{1 / 2}}<A(z) \quad \forall y \in Y .
$$

Then, we get

$$
\begin{aligned}
& \left(f(x, y)+\left(x^{\top} B x\right)^{1 / 2}\right)\left\{\sum_{i=1}^{s} t_{i}\left(h\left(z, y_{i}\right)-z^{\top} D v\right)\right\} \\
& -\left(h(x, y)-\left(x^{\top} D x\right)^{1 / 2}\right)\left\{\sum_{i=1}^{s} t_{i}\left(f\left(z, y_{i}\right)+z^{\top} B w\right)+\sum_{i=1}^{p} \mu_{j} g_{j}(z)\right\}<0,
\end{aligned}
$$

for all $y \in Y$. we get

If $y$ is replaced by $y_{i}$ in the above inequality and is multiplied by $t_{i}$, then summing up,

$$
\begin{aligned}
\sum_{i=1}^{s} t_{i}\left(f\left(x, y_{i}\right)+\left(x^{\top} B x\right)^{1 / 2}\right)\left\{\sum_{i=1}^{s} t_{i}\left(h\left(z, y_{i}\right)-z^{\top} D v\right)\right\} \\
-\left\{\sum_{i=1}^{s} t_{i}\left(h\left(x, y_{i}\right)-\left(x^{\top} D x\right)^{1 / 2}\right)\right\}\left\{\sum_{i=1}^{s} t_{i}\left(f\left(z, y_{i}\right)+z^{\top} B w\right)+\sum_{i=1}^{p} \mu_{j} g_{j}(z)\right\}<0 .
\end{aligned}
$$

By (2.6) and (6.3), we have

$$
\begin{aligned}
\Upsilon_{10}(x) \leq & \left\{\sum_{i=1}^{s} t_{i}\left(f\left(x, y_{i}\right)+\left(x^{\top} B x\right)^{1 / 2}\right)+\sum_{i=1}^{p} \mu_{j} g_{j}(x)\right\}\left\{\sum_{i=1}^{s} t_{i}\left(h\left(z, y_{i}\right)-z^{\top} D v\right)\right\} \\
& -\left\{\sum_{i=1}^{s} t_{i}\left(h\left(x, y_{i}\right)-\left(x^{\top} D x\right)^{1 / 2}\right)\right\}\left\{\sum_{i=1}^{s} t_{i}\left(f\left(z, y_{i}\right)+z^{\top} B w\right)+\sum_{i=1}^{p} \mu_{j} g_{j}(z)\right\} \\
< & \sum_{i=1}^{p} \mu_{j} g_{j}(x)\left\{\sum_{i=1}^{s} t_{i}\left(h\left(z, y_{i}\right)-z^{\top} D v\right)\right\} .
\end{aligned}
$$

Since $\sum_{i=1}^{s} t_{i}\left(h\left(z, y_{i}\right)-z^{\top} D v\right)>0$ and $\sum_{i=1}^{p} \mu_{j} g_{j}(x) \leq 0$, it follows that

$$
\Upsilon_{10}(x)<0=\Upsilon_{10}(z)
$$


Using the $B-(p, r)$-invexity with respect to $\eta$ and $b$ at $z$ of $\Upsilon_{10}(\cdot)$, we have the inequality

$$
\frac{1}{p} \nabla \Upsilon_{10}(z)\left(e^{p \eta(x, z)}-\mathbf{1}\right)<0
$$

This contradicts the equality of $(6.2) \times(1 / p)\left(e^{p \eta(x, z)}-\mathbf{1}\right)$. Here the proof is complete.

As a consequence of Theorems 6.1 and 6.2, we obtain Theorem 6.3. By a similar way, we can prove the strong duality and strictly converse duality theorems with respect to $(P)$ and (WD) which we state as follows.

Theorem 6.3 (strong duality). Let $x^{*}$ be an optimal solution of $(P)$ satisfying the hypothesis of Theorem 6.2. Then there exist $\left(s^{*}, t^{*}, y^{*}\right) \in K\left(x^{*}\right)$ and $\left(x^{*}, \mu^{*}, w^{*}, v^{*}\right) \in H_{3}\left(s^{*}, t^{*}, y^{*}\right)$ such that $\left(x^{*}, \mu^{*}, s^{*}, t^{*}, y^{*}, w^{*}, v^{*}\right)$ is feasible for (WD). If any of the conditions of Theorem 6.2 hold, then $\left(x^{*}, \mu^{*}, s^{*}, t^{*}, y^{*}, w^{*}, v^{*}\right)$ is an optimal solution of (WD) and the two problems $(P)$ and $(W D)$ have the same extremal values.

Theorem 6.4 (strict converse duality). Let $\bar{x}$ and $\left(z^{*}, \mu^{*}, s^{*}, t^{*}, y^{*}, w^{*}, v^{*}\right)$ be optimal solutions of $(P)$ and $(W D)$, respectively. Suppose that the assumptions of Theorem 6.3 are fulfilled and

$$
\begin{aligned}
\Upsilon_{11}(\cdot)= & {\left[\sum_{i=1}^{s^{*}} t_{i}^{*}\left(h\left(z^{*}, y_{i}^{*}\right)-\left(z^{*}\right)^{\top} D v^{*}\right)\right]\left[\sum_{i=1}^{s^{*}} t_{i}^{*}\left(f\left(\cdot, y_{i}^{*}\right)+(\cdot)^{\top} B w^{*}\right)+\sum_{j=1}^{p} \mu_{j}^{*} g_{j}(\cdot)\right] } \\
& -\left[\sum_{i=1}^{s^{*}} t_{i}^{*}\left(f\left(z^{*}, y_{i}^{*}\right)+\left(z^{*}\right)^{\top} B w^{*}\right)+\sum_{j=1}^{p} \mu_{j}^{*} g_{j}\left(z^{*}\right)\right]\left[\sum_{i=1}^{s} t_{i}^{*}\left(h\left(\cdot, y_{i}^{*}\right)-(\cdot)^{\top} D v^{*}\right)\right]
\end{aligned}
$$

is strictly $B-(p, r)$-invex with respect to $\eta$ and $b$ at $z^{*}$ on $X^{\circ} \cup p r_{X} \bar{\Gamma}$.

Then $\bar{x}=z^{*}$, that is, $z^{*}$ is an optimal solution of $(P)$ and $\sup _{y \in Y}\left(\left(f\left(z^{*}, y\right)+\right.\right.$ $\left.\left.\left(\left(z^{*}\right)^{\top} B z^{*}\right)^{1 / 2}\right) /\left(h\left(z^{*}, y\right)-\left(\left(z^{*}\right)^{\top} D z^{*}\right)^{1 / 2}\right)\right)=\mathcal{A}\left(z^{*}\right)$.

\section{Acknowledgment}

The author is partly supported by NSC, Taiwan

\section{References}

[1] T. Antczak, "Generalized fractional minimax programming with $B-(p, r)$-invexity," Computers and Mathematics with Applications, vol. 56, no. 6, pp. 1505-1525, 2008.

[2] H. C. Lai and J. C. Liu, "Minimax fractional programming involving generalised invex functions," ANZIAM Journal, vol. 44, no. 3, pp. 339-354, 2003.

[3] J. C. Liu and C. S. Wu, "On minimax fractional optimality conditions with invexity," Journal of Mathematical Analysis and Applications, vol. 219, no. 1, pp. 21-35, 1998.

[4] S. K. Mishra, S. Y. Wang, K. K. Lai, and J. M. Shi, "Nondifferentiable minimax fractional programming under generalized univexity," Journal of Computational and Applied Mathematics, vol. 158, no. 2, pp. 379395, 2003. 
[5] S. K. Mishra, R. P. Pant, and J. S. Rautela, "Generalized $\alpha$-invexity and nondifferentiable minimax fractional programming," Journal of Computational and Applied Mathematics, vol. 206, no. 1, pp. 122$135,2007$.

[6] G. J. Zalmai, "Optimality criteria and duality for a class of minimax programming problems with generalized invexity conditions," Utilitas Mathematica, vol. 32, pp. 35-57, 1987.

[7] C. R. Bector and B. L. Bhatia, "Sufficient optimality conditions and duality for aminimax problem," Utilitas Mathematica, vol. 27, pp. 229-247, 1985.

[8] S. Chandra and V. Kumar, "Duality in fractional minimax programming," Journal of the Australian Mathematical Society Series A, vol. 58, pp. 376-386, 1995.

[9] M. A. Hanson, "On sufficiency of the Kuhn-Tucker conditions," Journal of Mathematical Analysis and Applications, vol. 80, no. 2, pp. 545-550, 1981.

[10] H. C. Lai, J. C. Liu, and K. Tanaka, "Necessary and sufficient conditions for minimax fractional programming," Journal of Mathematical Analysis and Applications, vol. 230, no. 2, pp. 311-328, 1999.

[11] H. C. Lai and J. C. Lee, “On duality theorems for a nondifferentiable minimax fractional programming," Journal of Computational and Applied Mathematics, vol. 146, no. 1, pp. 115-126, 2002.

[12] H. C. Lai, J. C. Lee, and S. C. Ho, "Parametric duality on minimax programming involving generalized convexity in complex space," Journal of Mathematical Analysis and Applications, vol. 323, no. 2, pp. 1104 1115, 2006.

[13] S. K. Mishra, R. P. Pant, and J. S. Rautela, "Generalized $\alpha$-univexity and duality for nondifferentiable minimax fractional programming," Nonlinear Analysis, Theory, Methods and Applications, vol. 70, no. 1, pp. 144-158, 2009.

[14] W. E. Schmitendorf, "Necessary conditions and sufficient conditions for static minmax problems," Journal of Mathematical Analysis and Applications, vol. 57, no. 3, pp. 683-693, 1977.

[15] T. Antczak, "A class of $B$ - $(p, r)$-invex functions and mathematical programming," Journal of Mathematical Analysis and Applications, vol. 286, no. 1, pp. 187-206, 2003. 


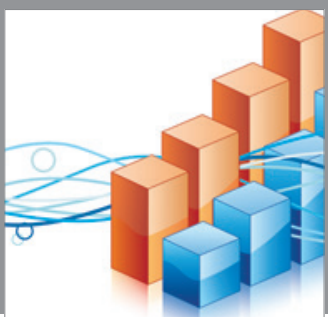

Advances in

Operations Research

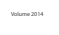

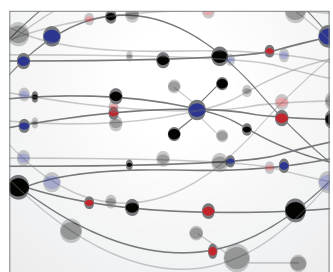

\section{The Scientific} World Journal
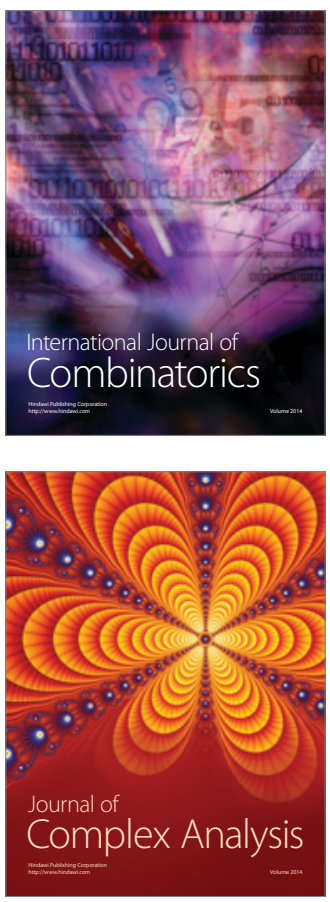

International Journal of

Mathematics and

Mathematical

Sciences
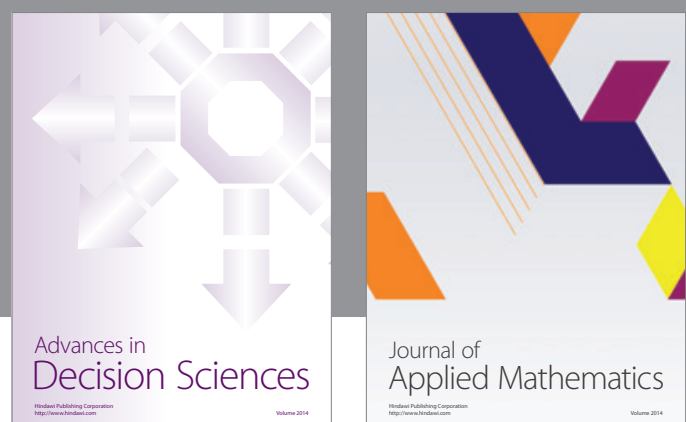

Journal of

Applied Mathematics
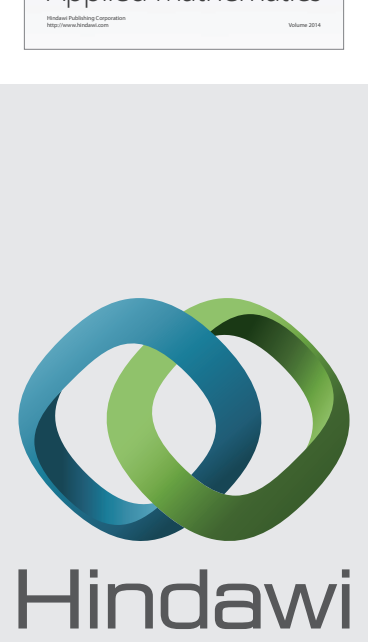

Submit your manuscripts at http://www.hindawi.com
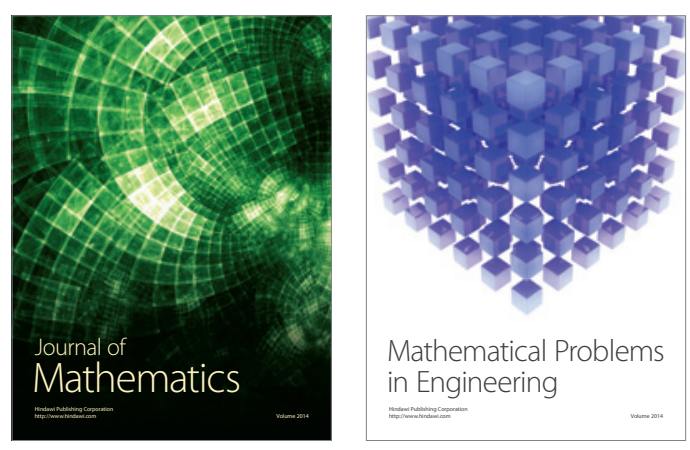

Mathematical Problems in Engineering
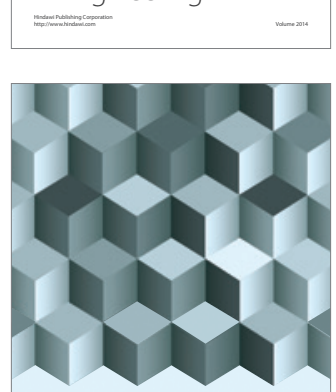

Journal of

Function Spaces
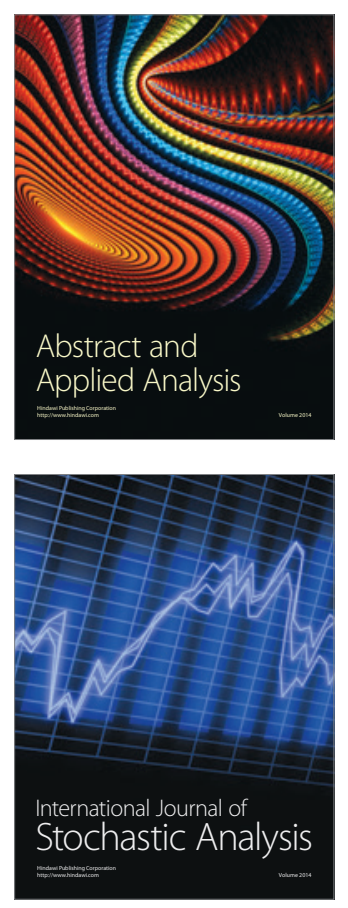

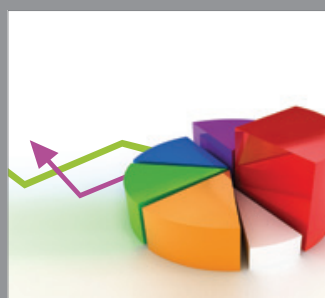

ournal of

Probability and Statistics

Promensencen
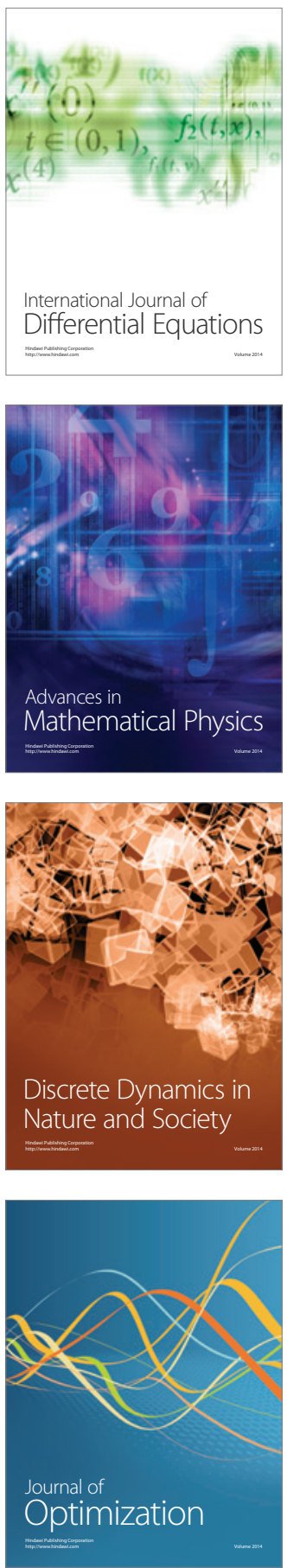